\title{
REVIEW \\ Compensatory angiogenesis and tumor refractoriness
}

\author{
RN Gacche
}

\begin{abstract}
Since the establishment of tumor angiogenesis as a therapeutic target, an excitement in developing the anti-angiogenic agents was resulted in tailoring a humanized monoclonal antibody (Bevacizumab) against vascular endothelial growth factor (VEGF): a key factor in recruiting angiogenesis. The past three decades' research in the area of angiogenesis also invented a series of novel and effective anti-angiogenic agents targeting the VEGF signaling axis. Despite the demonstrable clinical benefits of anti-angiogenic therapy, the preclinical and clinical data of the current therapeutic settings clearly indicate the transient efficacy, restoration of tumor progression and aggressive recurrence of tumor invasion after the withdrawal of anti-angiogenic therapy. Therefore, the impact of this therapeutic regime on improving overall survival of patients has been disappointing in clinic. The recent advances in pathophysiology of tumor angiogenesis and related molecular and cellular underpinnings attributed the conspiracy of compensatory angiogenic pathways in conferring evasive and intrinsic tumor resistance to anti-angiogenic agents. The understandings of how these pathways functionally cross-talk for sustaining tumor angiogenesis during VEGF blockade is essential and perhaps may act as a basic prerequisite for designing novel therapeutic strategies to combat the growing arrogance of tumors toward anti-angiogenic agents. The present review offers a discourse on major compensatory angiogenic pathways operating at cellular and molecular levels and their attributes with resistance to anti-angiogenic agents along with strategic opinions on future setting in targeting tumor angiogenesis.
\end{abstract}

Oncogenesis (2015) 4, e153; doi:10.1038/oncsis.2015.14; published online 1 June 2015

\section{INTRODUCTION}

Angiogenesis is a physiological process of formation of new capillaries on pre-existing vessels. The recent literature in the area of development of blood vessels is impressive in understanding the dynamics and complexities of vasculogenesis/angiogenesis. ${ }^{1}$ The process of angiogenesis appears to be fundamental for retrieving continues supply of oxygen and nutrients. The sprouting of new blood vessel initiates with dissolution of vascular basal membrane, increase in vascular permeability and degradation of extracellular matrix, followed by endothelial cell (EC) migration, invasion, proliferation and tube formation. The angiogenic switch refers to a consortium of several regulatory factors, which regulates angiogenesis by maintaining a strict balance between activators and inhibitors in normal physiological angiogenesis. The pathological angiogenesis is mostly a hallmark of cancer, wherein the developed vasculature is more complex, abnormal, leaky and torturous. ${ }^{2}$

In 1971, Judah Folkman, a pioneer researcher in tumor angiogenesis, first highlighted the significance of vasculature for the growth and proliferation of solid tumors. He demonstrated that if a tumor is deprived from generating its own blood supply, it would not grow more than $1-2 \mathrm{~mm}$ in size or it may wither and die. Since the opening of this research window, the preclinical and clinical data started accumulating in a logarithmic manner, with a clear intention of inhibiting the tumor angiogenesis. ${ }^{2}$ Till date, 10 anti-angiogenic agents have been approved by the US Food and Drug Administration: bevacizumab and ziv-aflibercept as antivascular endothelial growth factor (VEGF) agents, whereas sorafenib, sunitinib, pazopanib, axitinib, cabozantinib and regorafenib are approved as small-molecule RTK (receptor tyrosine kinase) inhibitors. ${ }^{3}$
Besides several appreciation reports describing the efficacy of anti-angiogenic agents in extending the survival of cancer patients by few months, ${ }^{4}$ the patients' benefit from the treatment is not satisfactory and is rather disappointing because of transient and modest performance of the anti-angiogenic agents in the clinic, offtarget toxicities and intrinsic refractoriness. ${ }^{2}$ Perhaps more serious is the aggressive invasion and expedite metastasis of tumors after withdrawal of anti-angiogenic drugs. ${ }^{5}$ Besides numerous clinical trials in progress and over dozens of molecules being engineered against VEGF/VEGF receptor (VEGFR) and non-VEGF pathways, ${ }^{4,6}$ the entire situation warrants the understanding of molecular underpinnings and loopholes supporting the bypass angiogenic mechanisms ensuring tumor progression and metastasis even after the treatment with effective anti-angiogenic agents.

\section{TUMORS EMPLOY MULTIPLE COMPENSATORY PRO-ANGIOGENIC FACTORS AND SIGNALING PATHWAYS IN ANTI-VEGF ENVIRONMENT}

In a broader sense, the multiple compensatory angiogenic factors/ signaling pathways that tumors employ during anti-VEGF stress (Figures 1and 2, ) can be conveniently categorized as VEGFdependent pathways, VEGF-independent signaling, mechnisms involving myeloid/stromal/tumor cell interactions and angiogenesis-independent vascular remodeling processes such as vessel cooption, intussusceptions and vascular mimicry.

VEGF-dependent compensatory angiogenic mechanisms The VEGF axis-dependent alteration pathways are initiated by the subtypes of VEGF such as VEGF-B, VEGF-C, VEGF-D and placenta growth factor (PIGF) (Figure 2). VEGF-B is observed to be

Tumor Biology Laboratory, School of Life Sciences, Swami Ramanand Teerth Marathwada University, Nanded, India. Correspondence: Professor RN Gacche, Tumor Biology Laboratory, School of Life Sciences, Swami Ramanand Teerth Marathwada University, Vishnupuri, Nanded 431 606, Maharashtra, India.

E-mail: rngacche@rediffmail.com

Received 17 March 2015; revised 4 April 2015; accepted 15 April 2015 


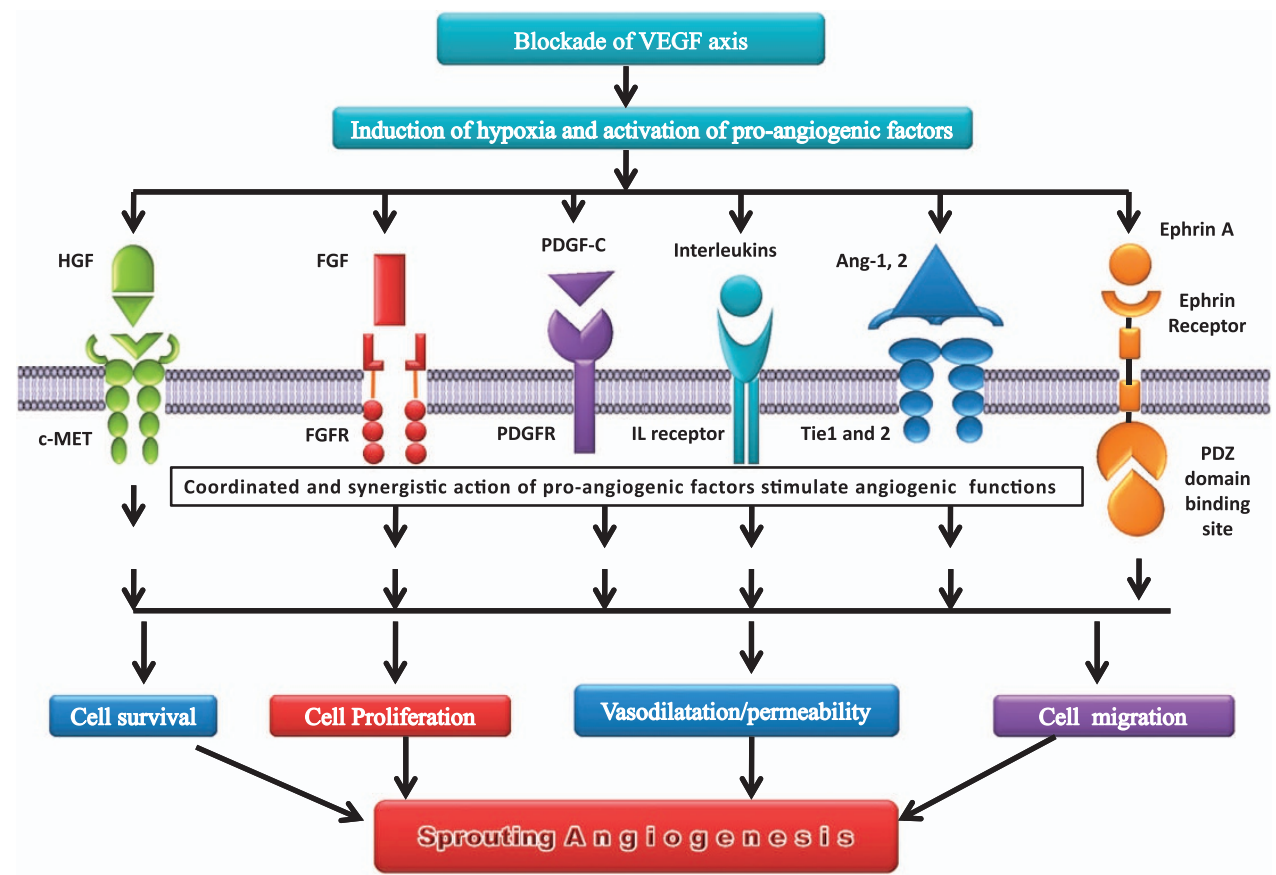

Figure 1. Role of pro-angiogenic factors in driving compensatory angiogenesis during blockade of VEGF axis.

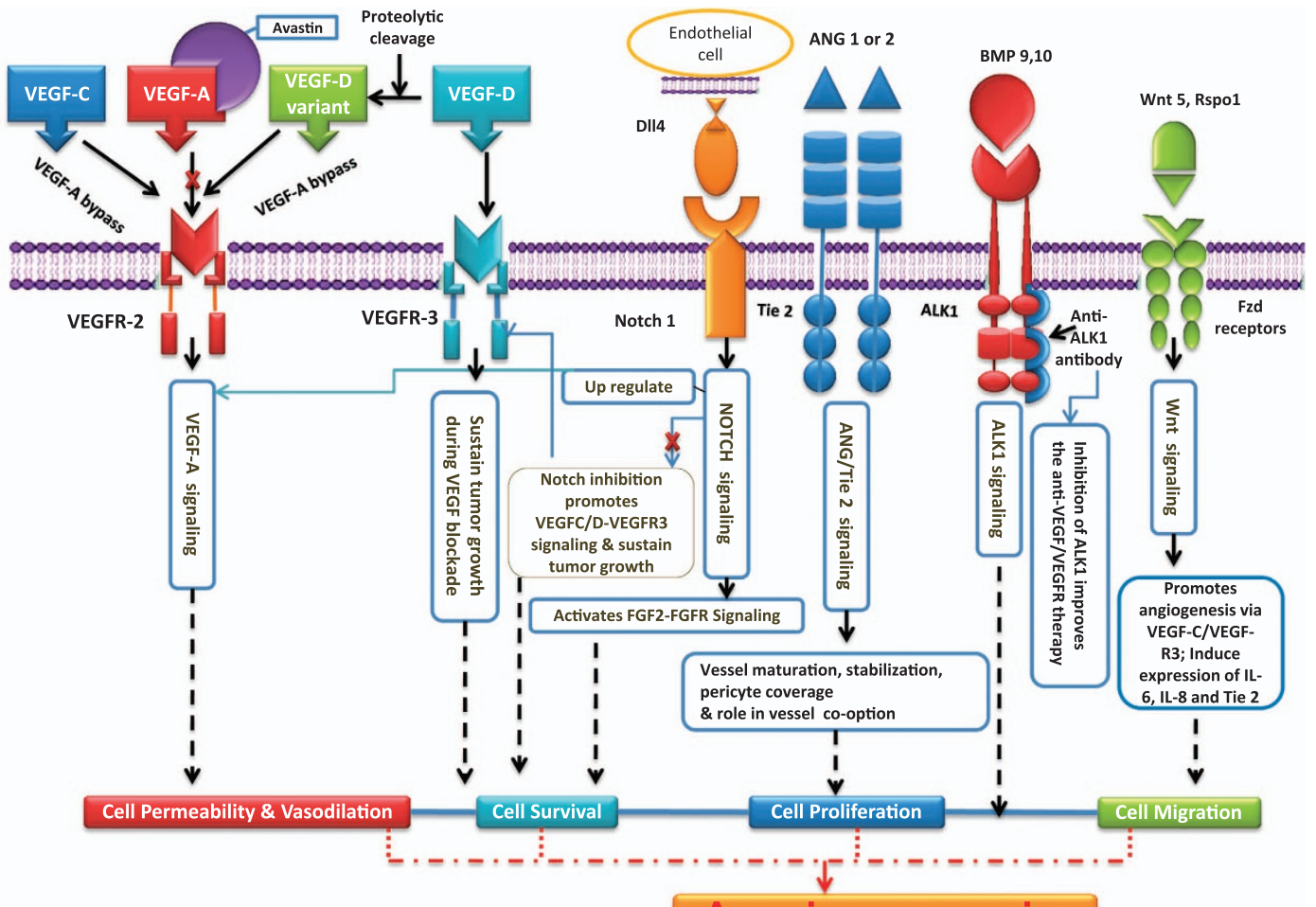

A nglogenes is

Figure 2. Compensatory angiogenic pathways.

upregulated in multiple malignancies having anticipatory role in supporting tumor cell migration.7 Ziv-aflibercept (Zaltrap, VEGFTrap) is an antibody linked to $\operatorname{lgG} 1$ backbone and possesses VEGFR1 and VEGFR2 as extracellular domains. Its binding preference is not only with VEGF-A but also with VEGF-B and PIGF. The role of VEGF-C and VEGF-D is primarily implicated in lympangiogenesis. ${ }^{8}$ It has been reported that a VEGF-C fragment generated through proteolytic cleavage has binding affinity with VEGFR-3 and perhaps could be an alternative bypass mechanism and a potential cause of resistance to anti-VEGF-A modalities. ${ }^{9}$ NRP-1 (VEGF receptor) not only binds to VEGF-A but also binds to PIGF. Moreover, NRP-2 interacts with VEGFR-2 and may enhance 
angiogenic signaling. Apart from VEGF-A, NRP-2 also binds with VEGF-C and PIGF, thereby having a significant role in angiogenesis. ${ }^{10}$ Clinical evidences also strengthen the possibility of involvement of PIGF in angiogenic rescue task, as upregulated levels of PIGF were detected in patients treated with anti-VEGFR therapy. ${ }^{11}$

\section{VEGF-independent compensatory pro-angiogenic factors and signaling mechanisms}

Clinical and experimental settings have identified several angiogenic growth factors having a key role in anti-VEGF escape mechanisms (Figure 1). Some of the key angiogenic factors involved in this puzzle of compensatory angiogenic signaling (Figure 2) includes fibroblast growth factors 1 and 2 (FGF1 and FGF2, respectively), ${ }^{12}$ hepatocyte growth factor/cMet pathway, ${ }^{13}$ angiopoietins, ${ }^{14}$ Delta4-Notch signaling pathway, ${ }_{1}^{15}$, plateletderived growth factor (PDGF)-C, ${ }^{16}$ interleukins, ${ }^{17}$ Ephrins, ${ }^{18}$ ALK1 signaling ${ }^{19}$ and Wnt signaling. ${ }^{20}$ FGF signaling has been considered as one of the major culprit for adaptive tumor resistance to VEGF-targeted inhibitors. ${ }^{12,21}$ In fact, binding of FGF receptors to FGF ligands causes activation of several signaling pathways such as phospholipase $C_{\gamma}$-protein kinase $C$, phosphatidylinositol 3-kinase (PI3K-AKT-mTOR), JAK/STAT (janus kinase-signal transducer and activator of transcription) and MAPK (mitogen-activated protein kinase: RAS/RAF/MAPK and RAS/MAPK/ERK). ${ }^{22}$ The signaling and cross-talk of these pathways (Figure 2) are involved in regulation of myriad of physiological processes including angiogenesis even after treating the tumors with VEGF-blocking agents. ${ }^{23}$ A clear evidence of evasive resistance mediated by FGF-dependent revascularization was observed in patients with recurrent glioblastoma taking treatment of cediranib (Recentin, Astra Zeneca, London, UK), a VEGFR inhibitor. Moreover, inhibition of FGF/VEGF using brivanib was found to be effective in treating mouse pancreatic neuroendocrine tumors demonstrating adaptive/evasive resistance to VEGF inhibition. ${ }^{24}$ Plethora of preclinical and clinical findings has highlighted the significance of Delta4Notch signalling as one of the pathways mediating the tumor refractoriness to anti-VEGF therapy. ${ }^{9,15,25,26}$ Inhibition of Delta4 leads to deregulation of angiogenesis, thereby resulting in excessive but non-functional vasculature, which can be conveniently used as an effective strategy for paralyzing the tumor growth that are relatively resistant to anti-VEGF therapeutic regime and for improving the therapeutic index of anti-VEGF agents by combining it with Delta4 inhibitors. ${ }^{26}$

In an investigation designed to understand the modes of tumor resistance to sunitinib, the HGF/c-Met pathway was identified as a culprit for anti-VEGF rescue mechanism. ${ }^{27}$ In clinical trial studies, in hepatocellular carcinoma (HCC) patients who were treated with VEGFR inhibitor, sorafenib, the progression-free survival was strongly correlated with low serum HGF levels as compared with patients with high serum HGF levels having progressive development of the disease. ${ }^{28}$ In recent cell coculture studies, a stromal cell (HSC-LX2) derived from the microenvironment of HCC was able to induce sorafenib resistance in HCC cells (Huh7) through multiple pathways including HGF/c-Met/Akt and Jak2/Stat3 (Chen et al. ${ }^{29}$ ).

The vessel normalization hypothesis claims that anti-VEGF agents, especially VEGFR2 blockers, normalize the vessels by proper covering of pericytes over the leaky vessels. This is a kind of vessel improvement and perhaps may act as an angiogenic escape mechanism. This proposed mechanism appears to be mediated by ANG1/Tie2 signaling. ${ }^{30}$ In case of MMTV-PyMT mammary carcinomas, the higher levels of ANG2 were associated with worse response to anti-angiogenic therapy containing bevacizumab. Nevertheless, there was lack of synergistic antiangiogenic or antitumor functions arising out of ANG2/VEGFR2 blockade. ${ }^{31}$ During VEGF arrest, administration of BowANG1 (an engineered construct that induces phosphorylation of Tie-2 in cultured ECs successfully prevented tumor regression by VEGF trap. $^{32}$ The major concerns of PDGFs in anti-VEGF rescue operations are largely associated with its role in activating pro-angiogenic stromal/perivascular cells conferring tumor resistance. ${ }^{9}$ In mouse lymphoma tumor model studies, the anti-VEGF-resistant tumor cells overexpressed PDGF-C mRNA and activated the adjacent tumor-associated fibroblasts (TAFs) to secrete PDGF-C, which in turn triggered tumor angiogenesis. ${ }^{16}$

Several members of interleukins (ILs) such as IL-1, IL-8, IL-12 (Vasudev and Reynolds) ${ }^{6}$ and, more recently, IL-17 (Chung et al. ${ }^{33}$ ) have been implicated in tumor refractoriness to anti-VEGF agents. A clear involvement of IL-8 has been reported in anti-VEGF tumor resistance in sunitinib-treated renal cell carcinoma. ${ }^{17}$ Chung et al. ${ }^{33}$ established a new link of IL-17-driven and stromal cellmediated signaling that confers resistance to anti-VEGF agents. Chung et al. $^{33}$ also showed that IL-17 signaling cascade mobilizes the granulocyte colony-stimulating factor (CSF)-dependent recruitment of $\mathrm{CD} 11 \mathrm{~b}^{+} \mathrm{Gr} 1^{+}$immature myeloid cells, which acts as major driving force for anti-VEGF tumor refractoriness. ${ }^{34}$ Mostly, the role of EphA2/EphrinA1 signaling has been attributed with compensatory angiogenesis and concerned tumor resistance. When the functions of VEGFR1 and R2 were blocked using antibodies, the resistance toward VEGFR2 blockade was observed in the form of revascularization and regrowth of tumors. The resistance to VEGF blockade was strongly correlated with hypoxiainduced upregulation of Ephrin A1 and other compensatory proangiogenic factors. ${ }^{35}$ In pancreatic tumors, overexpression of Eph A2 and Ephrin A1 was observed in VEGF-treated tumors and furthermore the elevated levels were correlated with tumor refractoriness. ${ }^{35,36}$

Suzuki et al. ${ }^{37}$ have demonstrated the pro-angiogenic effects of ALK1/BMP9 signaling, which includes proliferation of ECs, promotion of tumor angiogenesis in matrigel plug vascularization in a xenograft model of pancreatic cancer. In melanoma xenografts with upregulated human VEGF-A and resistance to RTK inhibitor, the combined treatment of bevacizumab in conjunction with PF-03446962 (a human lgG2 monoclonal anti-ALK1 antibody) significantly improved the efficacy of VEGF/VEGFR targeting agents, thus giving a clue of involvement of ALK1 signaling in bevacizumab resistance and thereby in promoting angiogenesis under VEGF blockade. ${ }^{38}$ The combination therapy of VEGFR tyrosine kinase inhibitor and ALK1 inhibitor (ALK1-FC) has also been assessed in VHL-deficient RCC murine xenograft models, where a clear evidence of resistance towards anti-VEGFR agents was observed. ${ }^{19}$ In zebrafish model studies, Rspo1/Wnt-mediated signaling promotes angiogenesis via VEGF-C/VEGF-R3, but not involving VEGF-A. The authors hope that the novel Rspo-WntVegfC-Vegfr3 pathway may have an important role as bypass angiogenic pathway during tumor angiogenesis. ${ }^{20}$

\section{Stromal/tumor cells recruited pro-angiogenic conspiracy and} tumor refractoriness

BMDCs: a reservoir of vascular progenitor cells. Bone marrowderived cells (BMDCs) constitute a major reservoir of vascular progenitor and vascular modulating cells, which infiltrate into stroma of tumors and have an important role in tumor progression including promotion of tumor angiogenesis independent of VEGF., 939 The most prominent cell populations of the BMDCs contributing to the tumor angiogenesis and progression (Figure 3) includes a lineage of $\mathrm{GR} 1^{+} \mathrm{CD} 11 \mathrm{~b}^{+}$myeloid progenitors, $\mathrm{CD} 11 \mathrm{~b}^{+} \mathrm{CD} 13^{+}$myeloid cells, CXCR4 ${ }^{+} \mathrm{VEGFR} 1^{+}$hemangiocytes, tumor-associated macrophages (TAMs) expressing CD11 $\mathrm{b}^{+} \mathrm{F} 4 / 80^{+}$, Tie2-expressing monocytes, $(\mathrm{PDGFR})^{+}$pericyte progenitors, a population of $\mathrm{CD} 45^{+} \mathrm{CD} 11 \mathrm{~b}^{+}$myeloid cells, tumor-infiltrated mast cells and neutrophils, and vascular endothelial-cadherin ${ }^{+} \mathrm{CD} 45^{+}$ vascular leukocytes and so on. ${ }^{39,40}$ Besides these prominent 


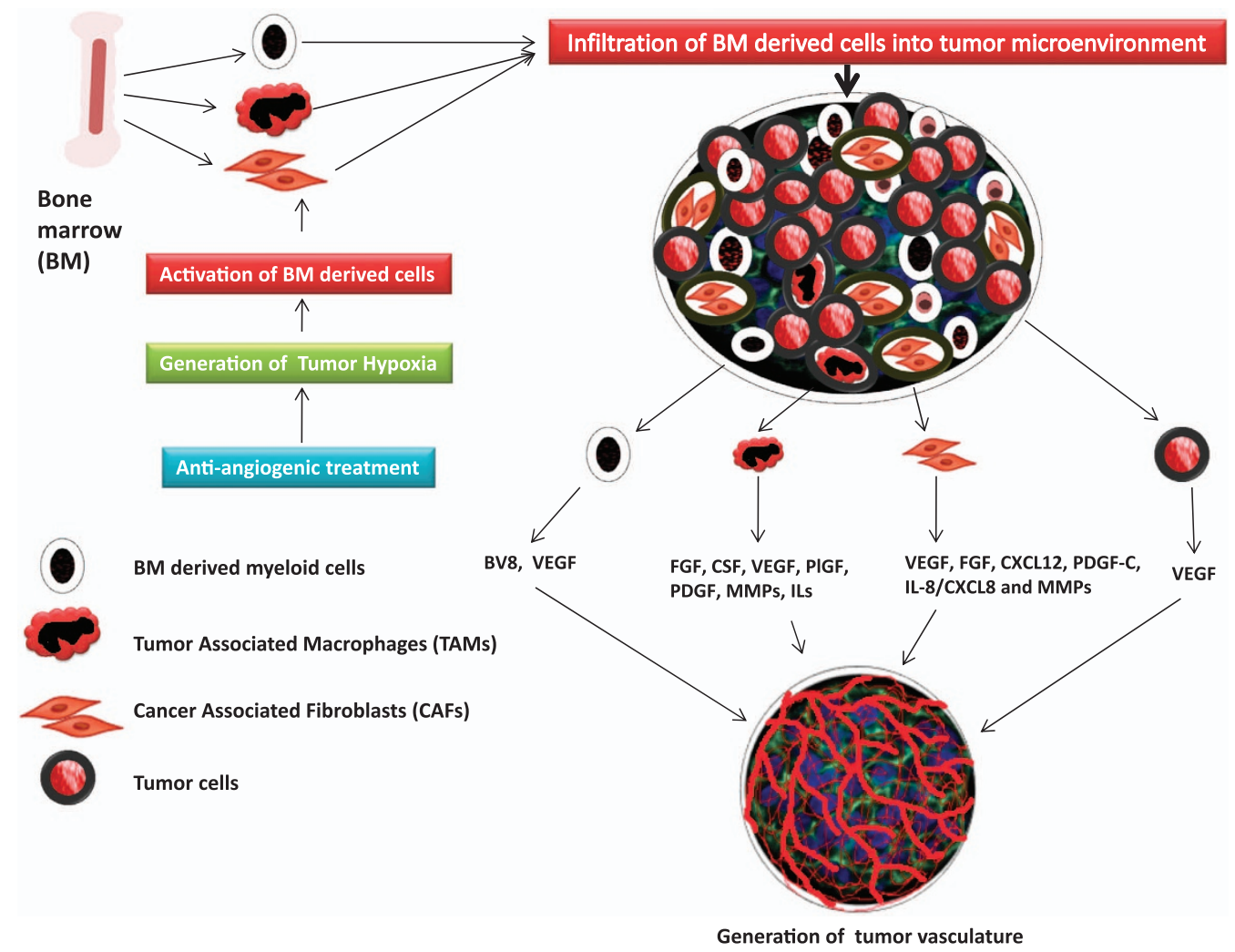

Figure 3. Role of stromal and tumor cells in compensatory angiogenesis.

pro-angiogenic BMDCs, several other lineages of myeloid cells involved in tumor progression and metastasis are extensively reviewed elsewhere. ${ }^{41,42}$ An investigation probing the mechanisms of tumor resistance toward the anti-angiogenic agents, a bone marrow-derived (BMD) $\mathrm{CD} 11 \mathrm{~b}^{+} \mathrm{Gr}^{+}$lineage encountered in anti-VEGF rescue mechanism, has been well documented. ${ }^{43}$ The $\mathrm{CD} 11 \mathrm{~b}^{+} \mathrm{Gr} 1^{+}$-driven angiogenesis was kind of co-ordinated consequence of a granulocyte-macrophage colony-stimulating factor, SDF-1a, placenta growth factor, granulocyte CSF and the granulocyte CSF-induced Bv8 (Bombina variagata peptide 8)secreted protein. ${ }^{43}$ In brief, the $\mathrm{CD} 11 \mathrm{~b}^{+} \mathrm{Gr} 1^{+}$-mediated angiogenesis is partly driven by the Bv8-dependent pathway, which is upregulated by granulocyte CSF that escapes VEGF and renders tumors more resistant to anti-VEGF agents. ${ }^{44}$

BMD TAMs have been considered as a primary cause of poor prognosis and resistance to anti-VEGF agents. ${ }^{45}$ TAM-mediated regulation of angiogenesis has been extensively investigated in animal tumor model studies. ${ }^{46,47}$ PLGF was overexpressed on antiVEGFR treatment; PLGF-mediated recruitment of pro-angiogenic and resistance-conferring TAMs might be a plausible mechanism for compensatory angiogenesis and tumor resistance. ${ }^{48}$ Nevertheless, the treatment of sorafenib in HCC tumors was also associated with elevated levels of CSF-1, SDF-1a and VEGF, which per se are chemokines for inviting macrophages. ${ }^{46}$ The finding of the HCC tumor model studies pinpoints the involvement of TAMs in tumor angiogenesis and progression under the treatment of sorafenib, which inhibits the VEGFR2, PDGF receptor and Raf kinases.

The current state-of-the-art literature cited elsewhere focus on several dimensions of TAF-mediated cues in tumor invasion, progression, angiogenesis and metastasis. ${ }^{49,50}$ The stroma and the invasive front of a tumor are usually occupied by clad of TAFs in different types of cancers such as lung, prostrate, breast, pancreas and colon. ${ }^{49}$ In particular, TAF-mediated conspiracy of driving angiogenesis under VEGF blockade is considered as a prominent cause of anti-VEGF tumor refractoriness (reviewed in Crawford and Ferrara, ${ }^{9}$ Ferrara $^{21}$ and Öhlund et al. ${ }^{51}$ ). Crawford et al. ${ }^{16}$ demonstrated that TAFs derived from resistant tumors sustained tumor growth and angiogenesis even after arresting the functions of VEGF. Further, it was found that TAF generated PDGF-C was a key factor in sustaining angiogenesis and tumor growth under anti-VEGF environment. The involvement of TAF-generated PDGF-C in tumor refractoriness was confirmed by arresting its functions using neutralizing antibody, which subsequently ameliorated the TAF-resistant induced angiogenesis and drastically delayed the growth of resistant tumors. ${ }^{16}$ di Tomaso et al..$^{52}$ also demonstrated that the stroma-generated PDGF-C overexpression was associated with resistance to anti-VEGF treatment in glioblastoma tumor model studies. $^{52}$

Pericytes and CSCs sustain tumors in anti-VEGF environment. Pericytes (peri: around; cyte: cell) are the contractile perivascular cells that wrap around the newly formed blood capillaries. It is now a well-established fact that pericytes have a significant role in pro-angiogenic signaling, especially in vascular morphogenesis, and have direct regulatory control over EC proliferation or quiescence. $^{53}$ Under hypoxic environment, the upregulated expression of VEGF-A in mature PCs recruit them to newly formed vessels. ${ }^{5}$ Pericytes also protect ECs through upregulation of $\mathrm{BCl}-\mathrm{w}$ protein, which protects ECs from apoptosis. ${ }^{54}$. Of note, antiangiogenic agents not only prune the neovasculature but also significantly induce apoptosis in ECs. ${ }^{54}$ Autocrine VEGF-A signaling in ECs in association with PCs offers an understanding of how PCs protects ECs under anti-angiogenic environment and recur angiogenesis after the relapse of anti-angiogenic therapy. Upregulation of PDGF-B in tumor cells has profound effect on increasing the PC coverage $\mathrm{e}^{55}$ and the reports state that PCs can protect ECs from VEGF withdrawal by activating compensatory pro-angiogenic signaling, especially PDGF receptor-medited angiogenic pathway in anti-VEGF therapy. ${ }^{55}$ In the current 
mainstream of tumor resistance, now it has been widely accepted that the anti-angiogenic drugs mainly prune ECs lacking PC coverage, while demonstrating limited efficacy on the PCs embarrassed mature vessels. ${ }^{56}$ Interestingly, low-to-moderate doses and transient treatment duration of anti-angiogenic agents have been shown to improve the efficacy of anti-angiogenic agents by a process of vessel normalization. ${ }^{3}$. Although the pro-angiogenic role of PCs is evolving, interestinlgy PCs derived from infantile hemangioma tumor of human demonstrated impressive pro-angiogenic activities and, of note, pro-angiogenic activity of these PCs was many fold higher than the retinal PCs. ${ }^{56}$

In the mainstream of cancer treatment, tumor heterogeneity has seeded several enquiries and imposed challenges in relation to its causes and consequences, concern with improving the drug efficacy and challenge of ameliorating the emerging drug resistance. ${ }^{57}$ Cancer stem cells (CSCs) represent a heterogenous subpopulation of cancer cells that retains the potential of selfrenewal, which leads to malignant progeny. Nevertheless, CSCs have also been identified as an emerging major driving force that governs tumor recurrence and confer resistance to anti-cancer agents. ${ }^{58}$ Putative CSCs have been reported in several tumor types including brain, melanoma, colorectal, breast, hepatic, head and neck, and prostate cancer. ${ }^{59}$ Several dimensions of angiogenic functions of CSCs have been recently reviewed elsewhere. ${ }^{59,60}$ The reason for suspecting CSCs in alternate angiogenesis and tumor resistance lies in their capabilities to produce much higher levels of VEGF in both anoxic and hypoxic environment than non-CSC population, ${ }^{60}$ which perhaps might be boosting strong angiogenic cues after the relapse of VEGF blockade. The report describes that the treatment of sunitinib and bevacizumab increases the number of breast CSCs by upregulation of hypoxia-inducible factor (HIF)- $1 a$ and through the activation of Wnt pathway via Akt/ $\beta$ catenin signaling. ${ }^{61}$

\section{HYPOXIA FUELS PRO-ANGIOGENIC STROMAL/TUMOR CELLS}

Tumor angiogenesis and hypoxia have been identified as hallmarks of solid tumors. HIF-1a mediates the transcription of genes involved in angiogenesis, oxygen consumption, migration and invasion of cancer cells. ${ }^{62}$ It has been described that antiangiogenic agents induce 'vascular regression,' which leads to increase in intratumor hypoxia and selection of more invasive metastatic clones of the cancer cells that are resistant to therapy including anti-angiogenic agents. ${ }^{63}$ Nevertheless, targeting hypoxia is being prioritized for overcoming the disappointing performance of anti-angiogenic agents in the clinic. ${ }^{62}$

A vast body of literature describes the role of hypoxia in activating and upregulating the compensatory angiogenic factors/ pathways and infiltrating different BMD pro-angiogenic cells including circulating endothelial progenitors and CSCs in tumor micro-environment. ${ }^{64}$. The most dramatic effect of hypoxia has been shown in recruiting BMDCs in TME, which later have an important role in compensatory angiogenesis. ${ }^{64,65}$ Blocking the functions of HIF impaired the mobilization of BMD pro-angiogenic cells such as VEGFR2 ${ }^{+} \mathrm{CD} 34^{+}$, VEGFR2 $^{+} \mathrm{CD} 117^{+}$and $\mathrm{CXCR} 4^{+} \mathrm{Sca} 1^{+}$ into the circulation and inhibition of tumor angiogenesis, thereby adversely affecting the tumor growth. ${ }^{66}$ Knockdown of Phd2 (a negative regulator of HIF-1a) in human colon cancer resulted in increasing the number of pro-angiogenic $\mathrm{CD}_{11} \mathrm{~b}^{+}$tumorassociated myeloid cells and promotion of vascularization. ${ }^{67}$ TAMs have been shown to preferentially accumulate in the hypoxic and necrotic regions in a variety of solid tumor cancers of humans such as breast, endometrium, ovary, bladder, colon and the oral cavity (reviewed in Quail and Joyce ${ }^{42}$ ). CSCs preferentially reside in hypoxic niches; moreover, they have upregulated levels of HIFs. ${ }^{68}$ The hypoxia induced by anti-angiogenic agents also mobilizes and increases the number of CSCs in breast cancer. ${ }^{61}$ Hu et al. ${ }^{69}$ have demonstrated that hypoxia-induced autophagy triggers tumor cell survival and adaptive resistance to anti-angiogenic therapy in the glioblastoma. Targeting the hypoxic microenvironment might serve effective anti-angiogenic combination therapies for combating the emerging resistance towards anti-angiogenic therapy. ${ }^{64}$

\section{ALTERNATIVE MECHANISMS OF ANGIOGENESIS- INDEPENDENT TUMOR VASCULATURE: AN OASIS DURING ANTI-VEGF STRESS}

The requirement of classic angiogenesis was previously thought to be a basic prerequisite for tumor progession and metastasis. Perhaps this assumption turned out as an illusion, as now sufficient evidence states that tumors can sustain their growth through various angiogenesis-independent mechanisms such as vessel cooption, vessel remodeling through intussusception, vascular mimicry (Figure 4) and other processes such as postnatal vasculogenesis, glomeruloid and looping angiogenesis. ${ }^{70}$ In vessel cooption, the tumor cells embrace the local blood vessels of the host during the invasion of tumors in surrounding host tissue and migrate along the vessels of the host organs. ${ }^{70,71}$ Vessel cooption

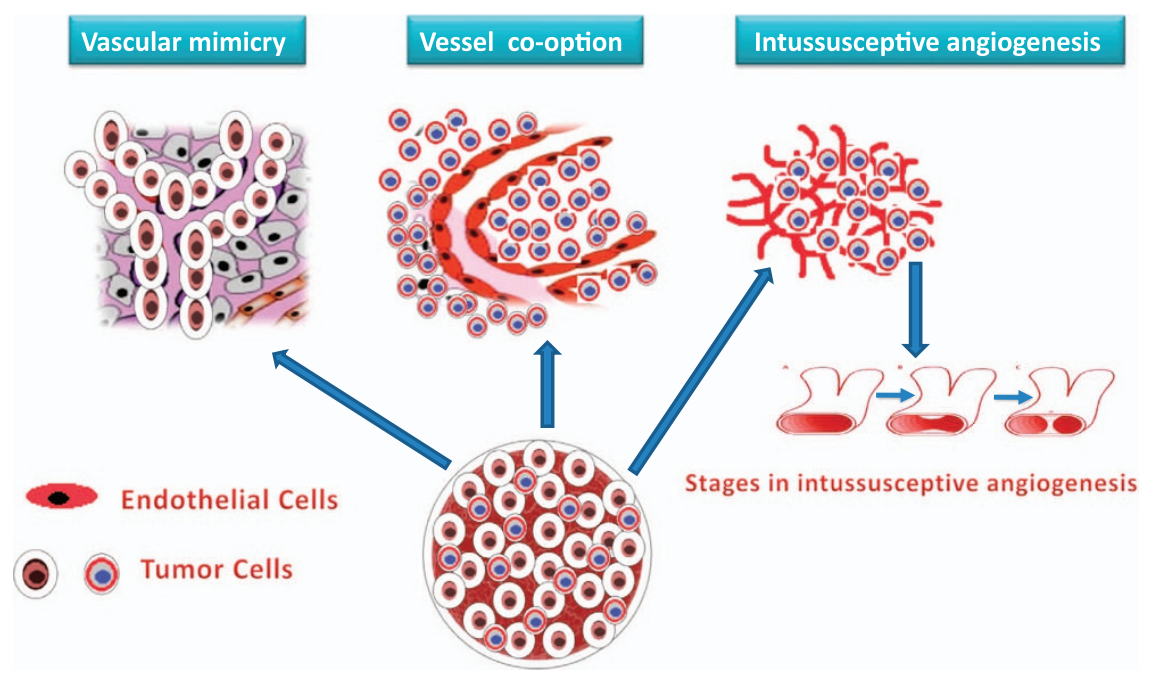

Figure 4. Angiogenesis-independent vascular remodeling mechanisms. 
is more frequently observed in cancers of densely vascularized organs such as the brain, lung and liver, wherein the primary tumor cells including metastases coopt with the adjacent existing quiescent blood vessels of the host tissue. ${ }^{72}$ Holash et al. ${ }^{70}$ proved that the implantation of $\mathrm{C} 6$ glioma cells in rat brain leads to formation of small vascularized tumors independent of angiogenesis. Kunkel et al. ${ }^{73}$ demonstrated that the systemic treatment with DC101, monoclonal antibody against VEGFR-2, increased cooption of quiescent cerebral vessels with residual tumors, which was further observed to have central cores of coopted vessels. The treatment of anti-angiogenic ZD6474 agent with brain metastases of cerebral melanoma was found to be associated with marked increase in vessel cooption. ${ }^{72}$ Interestingly, the coopted vessels respond differentially toward anti-angiogenic agents among different tumors. For example, liver metastases from breast cancer are more depended on cooption of the liver vasculature than that of colorectal origin. $^{74}$

The intussusceptive microvascular growth (IMG) also called splitting angiogenesis is a novel mode of vessel generation and vascular remodeling that might be acting as an anti-VEGF rescue meditor. IMG involves formation of two new vessels through fission of the pre-existing capillary plexus without sprouting. ${ }^{75}$ IMG, which happens to be an adaptive response to stress and hypoxia, is observed in several human tumors such as melanoma, colon, mammary carcinomas, B-cell non-Hodgkin's lymphoma and glioblastoma (reviewed in Patan et al. ${ }^{76}$ ). IMG has been identified as an important mechanism of rapid vascular remodeling in colon adenocarcinoma xenograft that contributes for intermittent blood flow in tumors. ${ }^{76}$ Treatment of mammary carcinoma allograft with VEGF tyrosine kinase inhibitor (PTK787/ZK222854) resulted in the development of extensive IMG in post-relapse period. ${ }^{77}$ In Lewis lung carcinoma and RIP-Tag 2 model studies, clear evidence of IMG and rapid revascularization was observed in tumors recovered after the treatment with VEGFR inhibitors. ${ }^{78}$ Perhaps, tumors might prefer IMG during anti-angiogenic exposure, as it is faster, and thermodynamically and metabolically more feasible as compared with sprouting angiogenesis. ${ }^{76,78}$

Maniotis et al. ${ }^{79}$ unraveled a tumor supporting crucial mechanism of generation of endothelium-independent vascular network called vasculogenic mimicry (VM). The authors demonstrated that the aggressive human melanoma cells mimic the functions of ECs and generate vascular tunnel-like phenotype that is foolproof in carrying red blood cells and plasma without involvement of ECs. ${ }^{79}$ VM has been reported in different human malignant tumours such as breast, melanoma, bladder, kidney, gliomas, glioblastomas, prostrate, ovarian, lung, sarcomas, cell renal cell carcinoma and astrocytoma. ${ }^{80}$ The imbroglio of VM is an amalgamation of three distinct elements, the primary one is the plasticity of aggressive tumor cells, followed by remodeling of the ECM and finally establishing the connections of the VM channels with the existing network of host microvessel system. ${ }^{81} \mathrm{VM}$ is critically regulated by several signaling pathways associated with embryonic/stem cell (Nodal and Notch4), vascular (VE-cadherin, VEGFR1, EphA2) and hypoxia-related HIF and Twist1 (Qiao et al. ${ }^{82}$ ). Clinical evidence of occurrence of VM in patients is strongly correlated with high risk of metastasis, poor prognosis, cancer recurrence and worse survival for patients of variety of cancers. ${ }^{81}$ Previous report entails the resistance of VM to anti-angiogenic inhibitors such as endostatin and TPN-470 in B16F10 murine melanoma model, as well as in melanoma tumor cells. ${ }^{83}$ It is interesting to note that hypoxia induces VM and, of note, CSCs, which possess tumor recurrence abilities, have a crucial role in $\mathrm{VM}^{84}$

\section{FUTURE SETTINGS IN ANTI-ANGIOGENIC THERAPY}

Before we endeavor for designing novel and effective strategies in anti-angiogenic therapy, it is important to prioritize the compensatory angiogenic mechanisms as targets for improving the therapeutic index of anti-angiogenic regime. Agents counteracting the redundant functioning of pro-angiogenic pathways and growth factors, patrolling the conspiracy of BMD tumor and stromal cells including pericytes and CSCs, antagonizing the angiogenesis-independent alternative mechanisms of vascular remodeling and overcoming the rebounds of invasiveness and metastasis after drug holidays should be an integral part of a judiciously formulated combinatorial therapeutic approach of future anti-angiogenic settings. ${ }^{85}$ Moreover, the systems pharmacology approaches integrating the biology of anti-VEGF resistance with the prognostic, predictive, pharmacodynamic and surrogate biomarkers coupled with computational imaging techniques such as dynamic contrast-enhanced magnetic resonance imaging and other imaging tools monitoring the vessel permeability, integrity and tumor perfusion might be helpful in ameliorating the drug resistance and optimizing the performance of anti-angiogenic drugs ${ }^{86}$.

\section{CONCLUSION}

Besides the emerging challenges, anti-angiogenic therapy has benefited not only for malignant diseases but also for nonmalignant human ailments as well. Before launching of the antiangiogenic therapy from the cradle to its action port, it was described as a therapy of 'resistant to resistance,' owing to the genetic stability of ECs, as anti-angigenic drugs mostly target ECs. ${ }^{2}$ However, this turned out to be an illusion with growing resistance toward anti-angiogenic agents. Sizable literature has accumulated in the recent past describing the fact that tumors employ multiple mechanisms of vascularization that compensates the treatment of the currently used anti-angiogenic agents. In the midst of emerging resistance to the currently available anti-angiogenic agents, future settings should capitalize more on developing novel anti-angiogenic agents targeting compensatory angiogenic mechanisms, which may be used in combination with currently available anti-angiogenic agents. In the current situation, combination therapeutic regimes seems to be a possible approach for amelioration of tumor resistance, as several preclinical and clinical studies have demonstrated significant impact of combination therapy in improving the clinical benefits to the patients.

\section{CONFLICT OF INTEREST}

The authors declare no conflict of interest.

\section{ACKNOWLEDGEMENTS}

I am thankful to UGC, New Delhi, for financial assistance (F. No. 42-196/2013(SR) and to Professor Pandit Vidyasagar, Vice Chancellor, SRTM University, for providing facilities and encouragement. Apologies to those authors whose excellent contributions could not be cited in this article on account of space constraint.

\section{REFERENCES}

1 Herbert SP, Stainier DYR. 2011. Molecular control of endothelial cell behaviour during blood vessel morphogenesis. Nat Rev Mol Cell Biol 2011; 12: 551-564.

2 Gacche RN, Meshram RJ. Angiogenic factors as potential drug target: efficacy and limitations of anti-angiogenic therapy. Biochemica Biophysica Acta 2014; 1846: 161-179.

3 Jain RK. Antiangiogenesis strategies revisited: from starving tumors to alleviating hypoxia. Cancer Cell 2014; 26: 605-622.

4 Ebos JML, Kerbel RS. Antiangiogenic therapy: impact on invasion, disease progression, and metastasis. Nat Rev Clin Oncol 2011; 8: 210-221.

5 Griffioen AW, Mans LA, de Graaf AM, Nowak-Sliwinska P, de Hoog CL, de Jong TA et al. Rapid angiogenesis onset after discontinuation of sunitinib treatment of renal cell carcinoma patients. Clin Cancer Res 2012; 18: 3961-3971.

6 Vasudev NS, Reynolds AR. Anti-angiogenic therapy for cancer: current progress, unresolved questions and future directions. Angiogenesis 2014; 17: 471-494.

7 Fischer C, Mazzone M, Jonckx B, Carmeliet P. FLT1 and its ligands VEGFB and PIGF: drug targets for antiangiogenic therapy? Nat Rev Cancer 2008; 8: 942-956. 
8 Lohela M, Bry M, Tammela T, Alitalo K. VEGFs and receptors involved in angiogenesis versus lymphangiogenesis. Curr Opin Cell Biol 2009; 21: 154-165.

9 Crawford Y, Ferrara N. Tumor and stromal pathways mediating refractoriness/ resistance to anti-angiogenic therapies. Trends Pharmacol Sci 2009; 30: 624-630.

10 Neufeld G, Kessler O. The semaphorins: versatile regulators of tumor progression and tumor angiogenesis. Nat Rev Cancer 2008; 8: 632-645.

11 Motzer RJ, Michaelson MD, Redman BG, Hudes GR, Wilding G, Figlin RA et al. Activity of SU11248, a multitargeted inhibitor of vascular endothelial growth factor receptor and platelet-derived growth factor receptor, in patients with metastatic renal cell carcinoma. J Clin Oncol 2006; 24: 16-24.

12 Welti JC, Gourlaouen M, Powles T, Kudahetti SC, Wilson P, Berney DM et al. Fibroblast growth factor 2 regulates endothelial cell sensitivity to sunitinib. Oncogene 2011; 30: 1183-1193.

13 Shojaei F, Lee JH, Simmons BH, Wong A, Esparza CO, Plumlee PA et al. HGF/c-Met acts as an alternative angiogenic pathway in sunitinib-resistant tumors. Cancer Res 2010; 70: 10090-10100.

14 Eklund L, Saharinen P. Angiopoietin signalling in the vasculature. Exp Cell Res 2013; 319: 1271-1280.

15 Li JL, Sainson RC, Oon CE, Turley H, Leek R, Sheldon H et al. DLL4-Notch signalling mediates tumor resistance to anti-VEGF therapy in vivo. Cancer Res 2011; 71: 6073-6083.

16 Crawford Y, Kasman I, Yu L, Zhong C, Wu X, Modrusan Z et al. PDGF-C mediates the angiogenic and tumorigenic properties of fibroblasts associated with tumors refractory to anti-VEGF treatment. Cancer Cell 2009; 15: 21-34.

17 Huang D, Ding Y, Zhou M, Rini BI, Petillo D, Qian CN et al. Interleukin-8 mediates resistance to antiangiogenic agent sunitinib in renal cell carcinoma. Cancer Res 2010; 70: 1063-1071.

18 Salvucci O, Tosato G. Essential roles of EphB receptors and EphrinB ligands in endothelial cell function and angiogenesis. Adv Cancer Res 2012; 114: 21-57.

19 Wang X, Solban N, Bhasin MK, Bahamon B, Zhang L, Signoretti S et al. ALK1-Fc inhibits tumor growth in a VEGF pathway resistance model of renal cell carcinoma. Cancer Res 2012; 72: LB-313.

20 Gore AV, Swift MR, Cha YR, Lo B, McKinney MC, Li W et al. Rspo1/Wnt signalling promotes angiogenesis via Vegfc/Vegfr3. Development 2011; 138: 4875-4886.

21 Ferrara N. Pathways mediating VEGF-independent tumor angiogenesis. Cytokine Growth Factor Rev 2010; 21: 21-26.

22 Turner N, Grose R. Fibroblast growth factor signalling: from development to cancer. Nat Rev Cancer 2010; 10: 116-129.

23 Cao Y, Cao R, Hedlund EM. Regulation of tumor angiogenesis and metastasis by FGF and PDGF signalling pathways. J Mol Med 2008; 86: 785-789.

24 Allen E, Walters IB, Hanahan D. Brivanib, a dual FGF/VEGF inhibitor, is active both first and second line against mouse pancreatic neuroendocrine tumors developing adaptive/evasive resistance to VEGF inhibition. Clin Cancer Res 2011; 17: 5299-5310.

25 Brzozowa M, Wojnicz R, Kowalczyk-Ziomek G, Helewski K. The Notch ligand Deltalike 4 (DLL4) as a target in angiogenesis-based cancer therapy? Contemp Oncol 2013; 17: 234-237.

26 Benedito R, Rocha SF, Woeste M, Zamykal M, Radtke F, Casanovas O et al. Notchdependent VEGFR3 upregulation allows angiogenesis without VEGF-VEGFR2 signalling. Nature 2012; 484: 110-114.

27 Shojaei F, Lee JH, Simmons BH, Wong A, Esparza CO, Plumlee PA et al. HGF/c-Met acts as an alternative angiogenic pathway in sunitinib-resistant tumors. Cancer Res 2010; 70: 10090-10100.

28 Miyahara K, Nouso K, Tomoda T, Kobayashi S, Hagihara H, Kuwaki K et al. Predicting the treatment effect of sorafenib using serum angiogenesis markers in patients with hepatocellular carcinoma. J Gastroenterol Hepatol 2011; 26: 1604-1611.

29 Chen W, Wu J, Shi H, Wang Z, Zhang G, Cao Y et al. Hepatic stellate cell coculture enables sorafenib resistance in Huh7 Cells through HGF/c-Met/Akt and Jak2/Stat3 pathways. Biomed Res Int 2014; 2014: 764981.

30 Goel S, Duda DG, Xu L, Munn LL, Boucher Y, Fukumura D et al. Normalization of the vasculature for treatment of cancer and other diseases. Physiol Rev 2011; 91 : 1071-1121.

31 Rigamonti N, Kadioglu E, Keklikoglou I, Wyser Rmili C, Leow CC, De Palma M. Role of angiopoietin-2 in adaptive tumor resistance to VEGF signalling blockade. Cell Rep 2014; 8: 696-706

32 Huang J, Bae JO, Tsai JP, Kadenhe-Chiweshe A, Papa J, Lee A et al. Angiopoietin-1/ Tie-2 activation contributes to vascular survival and tumor growth during VEGF blockade. Int J Oncol 2009; 34: 79-87.

33 Chung AS, Wu X, Zhuang G, Ngu H, Kasman I, Zhang J et al. An interleukin-17mediated paracrine network promotes tumor resistance to antiangiogenic therapy. Nat Med 2013; 19: 1114-1123.

34 Maniati E, Hagemann T. IL-17 mediates resistance to anti-VEGF therapy. Nat Med 2013; 19: 1092-1094.
35 Casanovas O, Hicklin DJ, Bergers G, Hanahan D. Drug resistance by evasion of antiangiogenic targeting of VEGF signalling in late-stage pancreatic islet tumors. Cancer Cell 2005; 8: 299-309.

36 Okazaki T, Ni A, Baluk P, Ayeni OA, Kearley J, Coyle AJ et al. Capillary defects and exaggerated inflammatory response in the airways of EphA2-deficient mice. Am J Pathol 2009; 174: 2388-2399.

37 Suzuki Y, Ohga N, Morishita Y, Hida K, Miyazono K, Watabe T. Bmp-9 induces proliferation of multiple types of endothelial cells in vitro and in vivo. J Cell Sci 2010; 123: 1684-1692

38 Hu-Lowe DD, Chen E, Zhang L, Watson KD, Mancuso P, Lappin P et al. Targeting activin receptor-like kinase 1 inhibits angiogenesis and tumorigenesis through a mechanism of action complementary to anti-VEGF therapies. Cancer Res 2011; 71: 1362-1373.

39 Ferrara N. Role of myeloid cells in vascular endothelial growth factor-independent tumor angiogenesis. Curr Opin Hematol 2010; 17: 219-224.

40 Rivera L, Pandika M, Bergers G. Escape mechanisms from antiangiogenic therapy: an immune cell's perspective. Adv Exp Med Biol 2014; 772: 83-99.

41 Murdoch C, Muthana M, Coffelt SB, Lewis CE. The role of myeloid cells in the promotion of tumor angiogenesis. Nat Rev Cancer 2008; 8: 618-631.

42 Quail DF, Joyce JA. Microenvironmental regulation of tumor progression and metastasis. Nat Med 2013; 19: 1423-1437.

43 Shojaei F, Wu X, Malik AK, Zhong C, Baldwin ME, Schanz S et al. Tumor refractoriness to anti-VEGF treatment is mediated by $\mathrm{CD} 11 \mathrm{~b}^{+} \mathrm{Gr} 1^{+}$myeloid cells. Nat Biotechnol 2007; 25: 911-920.

44 Shojaei F, Wu X, Qu X, Kowanetz M, Yu L, Tan M et al. G-CSF-initiated myeloid cell mobilization and angiogenesis mediate tumor refractoriness to anti-VEGF therapy in mouse models. Proc Natl Acad Sci USA 2009; 106: 6742-6747.

45 Noy R, Pollard JW. Tumor-associated macrophages: from mechanisms to therapy. Immunity 2014; 41: 49-61.

46 Guo C, Buranych A, Sarkar D, Fisher PB, Wang X-Y. The role of tumor-associated macrophages in tumor vascularisation. Vascular Cell 2013; 5: 1-12.

47 Hao NB, Lü MH, Fan YH, Cao YL, Zhang ZR, Yang SM. Macrophages in tumor microenvironments and the progression of tumors. Clin Dev Immunol 2012; 2012: 948098.

48 Fischer C, Jonckx B, Mazzone M, Zacchigna S, Loges S, Pattarini L et al. Anti-PIGF inhibits growth of VEGF(R)-inhibitor-resistant tumors without affecting healthy vessels. Cell 2007: 131: 463-475.

49 Kalluri R, Zeisberg M. Fibroblasts in cancer. Nat Rev Cancer 2006; 6: 392-401.

50 Kharaishvili G, Simkova D, Bouchalova K, Gachechiladze M, Narsia N, Bouchal J. The role of cancer-associated fibroblasts, solid stress and other microenvironmental factors in tumor progression and therapy resistance. Cancer Cell Int 2014; 14: 41.

51 Öhlund D, Elyada E, Tuveson D. Fibroblast heterogeneity in the cancer wound. J Exp Med 2014; 211: 1503-1523.

52 di Tomaso E, London N, Fuja D, Logie J, Tyrrell JA, Kamoun W et al. PDGF-C induces maturation of blood vessels in a model of glioblastoma and attenuates the response to anti-VEGF treatment. PLOS ONE 2009; 4: e5123.

53 Ding Y, Song N, Luo Y. Role of bone marrow-derived cells in angiogenesis: focus on macrophages and pericytes. Cancer Microenviron 2012; 5: 225-236.

54 Franco $M$, Roswall $\mathrm{P}$, Cortez E, Hanahan D, Pietras K. Pericytes promote endothelial cell survival through induction of autocrine VEGF-A signalling and Bcl-w expression. Blood 2011; 118: 2906-2917.

55 Song $N$, Huang $Y$, Shi H, Yuan S, Ding Y, Song X et al. Overexpression of plateletderived growth factor-BB increases tumor pericyte content via stromal-derived factor-1alpha/CXCR4 axis. Cancer Res 2009; 69: 6057-6064.

56 Bono AV, Pannellini T, Liberatore M, Montironi R, Cunico SC, Cheng L et al. Sorafenib's inhibition of prostate cancer growth in transgenic adenocarcinoma mouse prostate mice and its differential effects on endothelial and pericyte growth during tumor angiogenesis. Anal Quant Cytol Histol 2010; 32: 136-145.

57 Easwaran H, Tsai HC, Baylin SB. Cancer epigenetics: tumor heterogeneity, plasticity of stem-like states, and drug resistance. Mol Cell 2014; 54: 716-727.

58 Dick JE. Stem cell concepts renew cancer research. Blood 2008; 112: 4793-4807.

59 Juan MM-M, Dudley AC. Concise review: vascular stem cells and tumor angiogenesis. Stem Cells 2011; 29: 163-168.

60 Alvero AB, Fu HH, Holmberg J, Visintin I, Mor L, Marquina CC et al. Stem-like ovarian cancer cells can serve as tumor vascular progenitors. Stem Cells 2009; 27: 2405-2413.

61 Conley SJ, Gheordunescu E, Kakarala P, Newman B, Korkaya H, Heath AN et al. Antiangiogenic agents increase breast cancer stem cells via the generation of tumor hypoxia. Proc Natl Acad Sci 2012; 109: 2784-2789.

62 Rapisarda A, Melillo G. Overcoming disappointing results with antiangiogenic therapy by targeting hypoxia. Nat Rev Clin Oncol 2012; 9: 378-390.

63 Semenza GL. Hypoxia-inducible factors: mediators of cancer progression and targets for cancer therapy. Trends Pharmacol Sci 2012; 33: 207-214. 
64 Semenza GL. Cancer-stromal cell interactions mediated by hypoxia-inducible factors promote angiogenesis, lymphangiogenesis, and metastasis. Oncogene 2013; 32: 4057-4063.

65 Rapisarda A, Melillo G. Role of the hypoxic tumor microenvironment in the resistance to anti-angiogenic therapies. Drug Resist Updat 2009; 12: 74-80.

66 Lee K, Qian DZ, Rey S, Wei H, Liu JO, Semenza GL. Anthracycline chemotherapy inhibits HIF-1 transcriptional activity and tumor-induced mobilization of circulating angiogenic cells. Proc Natl Acad Sci USA 2009; 106: 2353-2358.

67 Chan DA, Kawahara TL, Sutphin PD, Chang HY, Chi JT, Giaccia AJ. Tumor vasculature is regulated by $\mathrm{PHD} 2$-mediated angiogenesis and bone marrow-derived cell recruitment. Cancer Cell 2009; 15: 527-538.

68 Vlashi E, McBride W, Pajonk F. Radiation responses of cancer stem cells. J Cell Biochem 2009; 108: 339-342.

$69 \mathrm{Hu}$ YL, DeLay M, Jahangiri A, Molinaro AM, Rose SD, Carbonell WS et al. Hypoxiainduced autophagy promotes tumor cell survival and adaptation to antiangiogenic treatment in glioblastoma. Cancer Res 2012; 72: 1773-1783.

70 Holash J, Maisonpierre PC, Compton D, Boland P, Alexander CR, Zagzag D et al. Vessel cooption, regression, and growth in tumors mediated by angiopoietins and VEGF. Science 1999; 284: 1994-1998.

71 Leenders WP, Küsters B, de Waal RM. Vessel co-option: how tumors obtain blood supply in the absence of sprouting angiogenesis. Endothelium 2002; 9: 83-87.

72 Leenders WP, Küsters B, Verrijp K, Maass C, Wesseling $P$, Heerschap A et al. Antiangiogenic therapy of cerebral melanoma metastases results in sustained tumor progression via vessel co-option. Clin Cancer Res 2004; 10: 6222-6230.

73 Kunkel P, Ulbricht U, Bohlen P, Brockmann MA, Fillbrandt R, Stavrou D et al. Inhibition of glioma angiogenesis and growth in vivo by systemic treatment with a monoclonal antibody against vascular endothelial growth factor receptor- 2 . Cancer Res 2001; 61: 6624-6628.

74 Stessels F, Van den Eynden G, Van der Auwera I, Salgado R, Van den Heuvel E, Harris AL et al. Breast adenocarcinoma liver metastases, in contrast to colorectal cancer liver metastases, display a non-angiogenic growth pattern that preserves the stroma and lacks hypoxia. Br J Cancer 2004; 90: 1429-1436.

75 Ribatti D, Djonov V. Intussusceptive microvascular growth in tumors. Cancer Lett 2012; 316: 126-131.

76 Patan S, Munn LL, Jain RK. Intussusceptive microvascular growth in a human colon adenocarcinoma xenograft: a novel mechanism of tumor angiogenesis. Microvasc Res 1996; 51: 260-272.
77 Hlushchuk R, Riesterer O, Baum O, Wood J, Gruber G, Pruschy M et al. Tumor recovery by angiogenic switch from sprouting to intussusceptive angiogenesis after treatment with PTK787/ZK222584 or ionizing radiation. Am J Pathol 2008; 173: $1173-1185$.

78 Mancuso MR, Davis R, Norberg SM, O'Brien S, Sennino B, Nakahara T et al. Rapid vascular regrowth in tumors after reversal of VEGF inhibition. J Clin Invest 2006; 116: $2610-2621$

79 Maniotis AJ, Folberg R, Hess A, Seftor EA, Gardner LM, Pe'er J et al. Vascular channel formation by human melanoma cells in vivo and in vitro: vasculogenic mimicry. Am J Pathol 1999; 155: 739-752.

80 Kirschmann DA, Seftor EA, Hardy KM, Seftor RE, Hendrix MJ. Molecular pathways: vasculogenic mimicry in tumor cells: diagnostic and therapeutic implications. Clin Cancer Res 2012; 18: 2726-2732.

81 Fan $Y Z$, Sun W. Molecular regulation of vasculogenic mimicry in tumors and potential tumor-target therapy. World J Gastrointest Surg 2010; 2: 117-127.

82 Qiao L, Liang N, Zhang J, Xie J, Liu F, Xu D et al. Advanced research on vasculogenic mimicry in cancer. J Cell Mol Med 2015; 19: 315-326.

83 van der Schaft DW, Seftor RE, Seftor EA, Hess AR, Gruman LM, Kirschmann DA et al. Effects of angiogenesis inhibitors on vascular network formation by human endothelial and melanoma cells. J Natl Cancer Inst 2004; 96: 1473-1477.

84 Liu TJ, Sun BC, Zhao XL, Zhao XM, Sun T, Gu Q et al. CD133+ cells with cancer stem cell characteristics associates with vasculogenic mimicry in triple-negative breast cancer. Oncogene 2013; 32: 544-553.

85 van Beijnum JR, Nowak-Sliwinska P, Huijbers EJ, Thijssen VL, Griffioen AW. The great escape; the hallmarks of resistance to antiangiogenic therapy. Pharmacol Rev 2015; 67: 441-446.

86 Sharan S, Woo S. Systems pharmacology approaches for optimization of antiangiogenic therapies: challenges and opportunities. Front Pharmacol 2015; 6: 33

(i) Oncogenesis is an open-access journal published by Nature Publishing cc) Group. This work is licensed under a Creative Commons Attribution 4.0 International License. The images or other third party material in this article are included in the article's Creative Commons license, unless indicated otherwise in the credit line; if the material is not included under the Creative Commons license, users will need to obtain permission from the license holder to reproduce the material. To view a copy of this license, visit http://creativecommons.org/licenses/by/4.0/ 